\title{
Detection of large magnetoanisotropy of electron spin dephasing in a high-mobility two-dimensional electron system in a [001] $\mathrm{GaAs} / \mathrm{Al}_{x} \mathrm{Ga}_{1-x} \mathrm{As}$ quantum well
}

\author{
D. Stich, ${ }^{1}$ J. H. Jiang, ${ }^{2}$ T. Korn, ${ }^{1}$ R. Schulz, ${ }^{1}$ D. Schuh,,${ }^{1}$ W. Wegscheider, ${ }^{1}$ M. W. Wu, ${ }^{2, *}$ and C. Schüller ${ }^{1, \dagger}$ \\ ${ }^{1}$ Institut für Experimentelle und Angewandte Physik, Universität Regensburg, D-93040 Regensburg, Germany \\ ${ }^{2}$ Hefei National Laboratory for Physical Sciences at Microscale and Department of Physics, University of Science and Technology of \\ China, Hefei, Anhui 230026, China
}

(Received 9 May 2007; revised manuscript received 16 July 2007; published 14 August 2007)

\begin{abstract}
In time-resolved Faraday rotation experiments, we have detected an in-plane anisotropy of the electron spin-dephasing time (SDT) in an $n$-modulation-doped $\mathrm{GaAs} / \mathrm{Al}_{0.3} \mathrm{Ga}_{0.7} \mathrm{As}$ single quantum well. The SDT was measured with magnetic fields of $B \leqslant 1 \mathrm{~T}$, applied in the [110] and [1 $\overline{10}$ ] in-plane crystal directions of the GaAs quantum well. For fields along [1히, we have found up to a factor of about 2 larger SDT than in the perpendicular direction. The observed SDTs also show strong anisotropic magnetic-field dependence. Fully microscopic calculations, by numerically solving the kinetic spin Bloch equations considering the D'yakonovPerel' and the Bir-Aronov-Pikus mechanisms, reproduce the experimental findings quantitatively. This quantitative analysis of the data allowed us to determine the relative strengths of Rashba and Dresselhaus terms in our sample. Moreover, we could predict the SDT for spins aligned in the [110] in-plane direction to be on the order of several nanoseconds, which is up to 2 orders of magnitude larger than that in the perpendicular in-plane direction.
\end{abstract}

DOI: 10.1103/PhysRevB.76.073309

PACS number(s): 73.20.-r, 85.75.-d, 71.70.Ej

The exploration of electron spin relaxation and dephasing is at the heart of the research of semiconductor spintronics. ${ }^{1,2}$ For potential applications in quantum computation or spin transistor devices, ${ }^{3}$ the investigation and knowledge about the relevant spin-dephasing channels are important prerequisites. The most powerful techniques for the study of spin dynamics in semiconductors are all-optical techniques, such as time-resolved photoluminescence or time-resolved Kerr or Faraday rotation (TRFR), where the latter are so-called pump-probe techniques. In fact, TRFR experiments have been used to detect extremely long spin relaxation times in bulk GaAs structures, which were $n$-type doped, close to the metal-to-insulator transition. ${ }^{4}$ So far, however, comparatively little attention has been paid to the experimental investigation of spin dynamics in quantum wells containing free electrons (e.g., in Refs. 5-7). In the majority of experiments, spin aligned charge carriers are created via the absorption of circularly polarized light. Hence, the direction of optically oriented spins is parallel or antiparallel to the direction of light propagation. Therefore, in typical time-resolved experiments on quantum-well samples with normal incidence, the spins of the photoexcited carriers point perpendicular to the quantumwell plane, i.e., in the growth direction of the layered sample. In a pioneering experiment, Ohno et al. ${ }^{8}$ and Döhrmann et $a l .{ }^{9}$ confirmed experimentally in quantum-well samples, which were grown in the [110] crystal direction, a very long spin relaxation time on the order of nanoseconds for spins, aligned parallel to the growth direction. This was predicted theoretically to be due to the absence of the D'yakonovPerel' spin relaxation mechanism ${ }^{10}$ in this particular crystal direction. These discoveries lead to a growing interest in [110] structures, which promised to be well-suited candidates for spintronic devices. There are, however, essentially two important reasons which might suggest using standard [001]grown heterostructures for spin-injection devices: (i) In typical real transport devices for spin injection and manipulation, as, e.g., motivated by the Datta-Das spin transistor, ${ }^{3}$ layered ferromagnetic electrodes are employed as spin injectors, which exhibit in-plane magnetization directions, i.e., the injected spins are typically in-plane with respect to the semiconductor structure. (ii) Due to the lower growth temperature for the growth on [110] substrates, the mobility in modulation-doped heterostructures, grown in that crystal direction, is generally lower than that in standard [001] structures.

Quite some time ago, it was demonstrated theoretically that also in [001] semiconductor heterostructures of zincblend type, spin relaxation could be greatly suppressed for spins pointing into the [110] in-plane direction. ${ }^{13} \mathrm{~A}$ giant spin relaxation anisotropy was predicted for in-plane spin orientation, where spin relaxation should be strong in [1 $\overline{1} 0]$ direction and weak in the perpendicular [110] direction. The strength of the anisotropy roughly depends on the ratio between the Rashba ${ }^{11}$ and Dresselhaus ${ }^{12}$ spin-orbit coupling terms: The anisotropy should be maximal if both terms have equal strengths. ${ }^{14}$ For this case, Schliemann et al. proposed a spin transistor device which uses diffusive transport. ${ }^{15} \mathrm{Re}-$ cently, Averkiev et al. performed polarization-resolved cw photoluminescence measurements on a nominally undoped [001]-grown quantum well at liquid nitrogen temperature, ${ }^{16}$ determining the depolarization of the photoluminescence in an applied in-plane field (Hanle effect). From the experimental data, the authors extracted in-plane spin relaxation times, which differed by about a factor of 2 for the two perpendicular in-plane directions, which showed evidence for the inplane anisotropy of the spin-dephasing time (SDT). More recently, Liu et al. ${ }^{22}$ performed Kerr rotation measurement on a [001]-grown two-dimensional electron gas at $150 \mathrm{~K}$ and extracted the in-plane SDTs along different directions with a factor of 1.3. In this work, we report TRFR experiments on a high-mobility two-dimensional electron system (2DES) with in-plane magnetic fields. We could detect a magnetoanisot- 
(a)
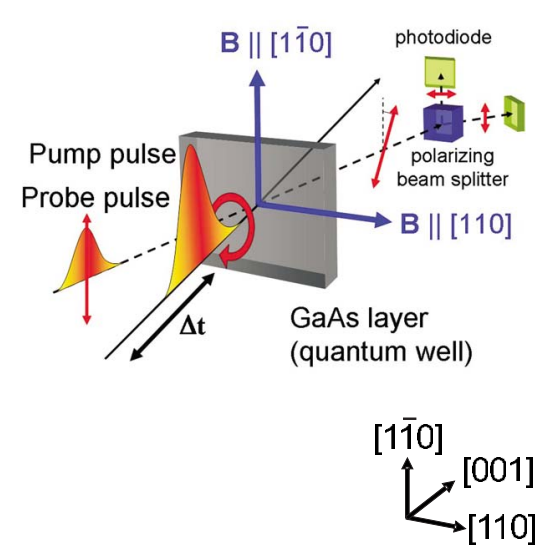

(b)

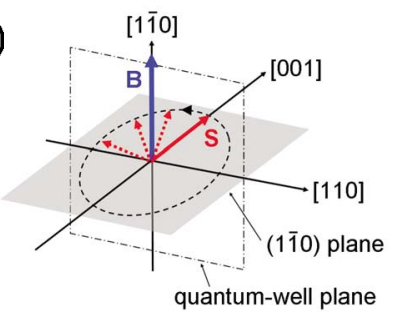

(c)

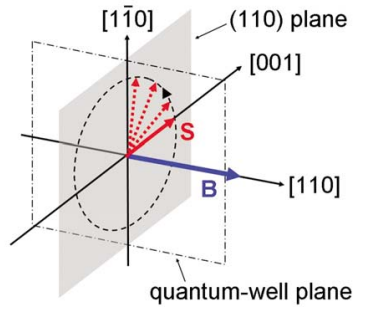

FIG. 1. (Color online) (a) Schematic picture of the timeresolved Faraday rotation experiment. In-plane magnetic fields are applied either in the [110] or the [1] 10$]$ directions. (b) Sketch of the precession of optically oriented spins about a $[1 \overline{1} 0]$ in-plane magnetic field. (c) Same as (b) but for [110] magnetic field. ropy of the SDT, measured via the out-of-plane component of the initial spin polarization. Calculations employing the fully microscopic kinetic spin Bloch equations, ${ }^{17-21}$ taking into account all relevant spin relaxation mechanisms (including both the D'yakonov-Perel' and the Bir-Aronov-Pikus mechanisms $\left.{ }^{23,24}\right)$, reproduce the experimental data quantitatively without any parameter for the SDT. This allows us to estimate the maximum in-plane SDT in our standard highmobility quantum-well structure to be on the order of several nanoseconds, which is about 2 orders of magnitude larger than in the perpendicular in-plane direction.

Our sample is a 20-nm-wide, one-sided modulation-doped GaAs- $\mathrm{Al}_{0.3} \mathrm{Ga}_{0.7} \mathrm{As}$ single quantum well. The electron density and mobility at $T=4.2 \mathrm{~K}$ are $n_{e}=2.1 \times 10^{11} \mathrm{~cm}^{-2}$ and $\mu_{e}=1.6 \times 10^{6} \mathrm{~cm}^{2} / \mathrm{V} \mathrm{s}$, respectively. For measurements in transmission geometry, the sample was glued onto a glass substrate with an optical adhesive, and the substrate and buffer layers were removed by selective etching. The sample was mounted in the ${ }^{3} \mathrm{He}$ insert of a superconducting split-coil magnet cryostat. All measurements were taken at a temperature of $T=4.5 \mathrm{~K}$. For the TRFR measurements, two laser beams from a mode-locked Ti:sapphire laser, which is operated at $80 \mathrm{MHz}$ repetition rate, were used. The laser pulses had a temporal length of about $600 \mathrm{fs}$ each, resulting in a spectral width of about 3-4 meV, which allowed for a resonant excitation. The laser wavelength was tuned to excite electrons from the valence band to states slightly above the Fermi energy of the host electrons in the conduction band. Both laser beams were focused to a spot of approximately $60 \mu \mathrm{m}$ diameter on the sample surface. The pump pulses were circularly polarized in order to create spin-oriented electrons in the conduction band, with spins aligned perpendicular to the quantum-well plane, i.e., in the [001] direction. The pump power was set to excite an initial spin polarization of the 2DES of about $6 \%$. Such a high spin polarization ensures a sufficiently long spin-dephasing time to subtract the spin-dephasing time from the full spin precession at low magnetic field. ${ }^{18,25}$ The rotation of the linear polarization of the time-delayed probe pulse, due to the Faraday effect, was measured by an optical bridge [see Fig. 1(a)]. Due to its near-normal incidence on the sample, the polarization rota- tion of the probe pulse is caused by the out-of-plane component of the spin polarization of the $2 \mathrm{DES}$.

Figure 1(a) is a schematic of the experiment, showing the orientation of the sample, relative to the laser beams and the magnetic fields. Figures 1(b) and 1(c) illustrate the precessional motion of the spin polarization in the two cases investigated in this Brief Report: For a magnetic field applied parallel to the $[1 \overline{1} 0]$ ([110]) direction [Fig. 1(b)] ([Fig. 1(c)]), the spins, which are created with an out-of-plane orientation parallel to the [001] direction, are forced to precess within the $(1 \overline{10})[(110)]$ plane. Besides the magnetic field, the spins also precess around the momentum k-dependent effective magnetic field due to the Dresselhaus and Rashba spin-orbit couplings, which provide an inhomogeneous broadening leading to the spin dephasing. ${ }^{14,17-21}$ Spins, precessing within the $(1 \overline{10})[(110)]$ plane, feel the inhomogeneous broadening along the $[1 \overline{1} 0][(110)]$ direction, which reads $(\alpha-\beta) k_{x}$ $\left[-(\alpha+\beta) k_{y}\right]$, if the $y(x)$ axis is along $[1 \overline{1} 0]([110]) .{ }^{14}$ Here, $\alpha(\beta)$ represents the Rashba (Dresselhaus) coefficient. Strong anisotropy is predicted when $\alpha$ is comparable to $\beta$. Thus, during their precessional motion, the spins are expected to probe the relaxation times in the different in-plane directions. In this work, we demonstrate that the effect on their overall, averaged SDT can be observed by measuring the out-ofplane component of the spin polarization.

Figure 2 shows TRFR traces taken for various magneticfield amplitudes applied parallel to the [1힐 direction (black traces), which show an exponentially damped oscillation of the out-of-plane component of the spin polarization. The temporal decay of the signal is determined by the SDT. A TRFR trace taken for a magnetic field of $0.9 \mathrm{~T}$ parallel to [110] (red trace) is shown directly above for comparison. By comparing it to the corresponding trace below, the dephasing anisotropy is clearly visible. Figure 3(a) shows the SDTs extracted from the experimental data by fitting an exponentially damped cosine function to the TRFR traces. The experimentally determined times are in excellent agreement with the calculations. A rather striking feature is the minimum in the SDT observed in Fig. 3(a) for a magnetic field of 


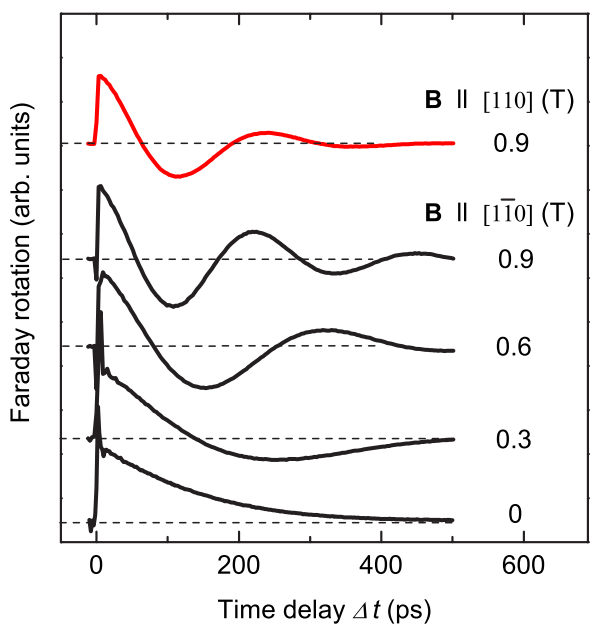

FIG. 2. (Color online) Experimental TRFR traces for different directions of the in-plane magnetic field.

$0.2 \mathrm{~T}$, which is clearly present in both the experiment and the calculations. The corresponding experimental trace is shown in Fig. 3(c), while Fig. 3(b) shows the trace at $0.2 \mathrm{~T}$ for the perpendicular magnetic-field direction. One can clearly see that in the latter situation, the signal, and hence the out-of-plane component of the spin polarization, reflects more than half a precession cycle of the spin polarization during the measured time window, while in the former case [Fig. 3(c)], the spin polarization is completely relaxed within a quarter cycle. Another feature is that there is a maximum of the SDT at $B=0.5 \mathrm{~T}$ for a magnetic field along the [1 $\overline{1} 0]$ direction. The strength of the Rashba term, $\alpha=0.65 \beta$, in the calculations is tuned to generate the best fit to the experimental data, whereas the strength of the Dresselhaus term, $\beta$ $=1.38 \mathrm{meV} \AA$, is determined following Ref. $20 .{ }^{26}$ The
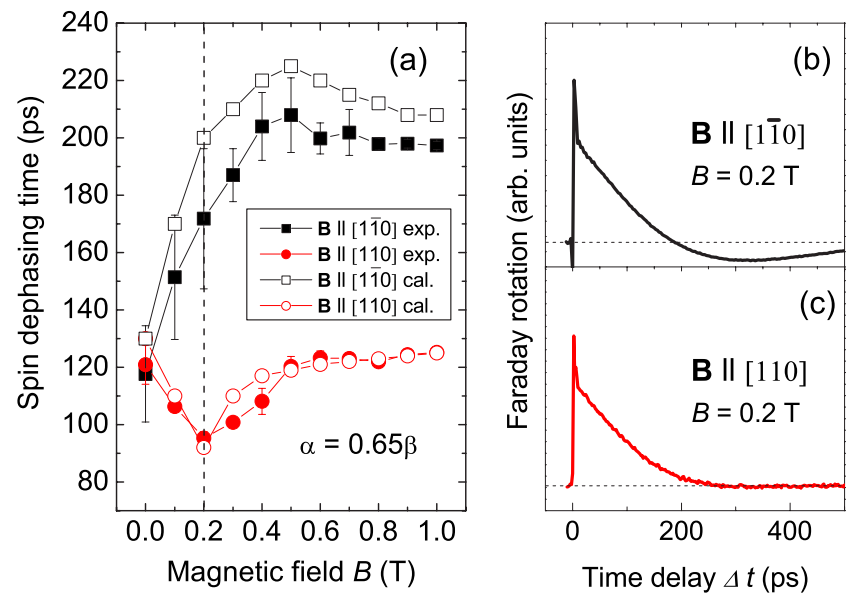

FIG. 3. (Color online) (a) Comparison of the experimental (solid symbols) and theoretically calculated (open symbols) spindephasing times for different in-plane magnetic-field directions. $\alpha$ and $\beta$ are the Rashba and Dresselhaus spin-orbit coefficients, respectively. [(b) and (c)] Comparison of experimental TRFR traces at $B=0.2 \mathrm{~T}$ for the two different in-plane directions of the magnetic field.

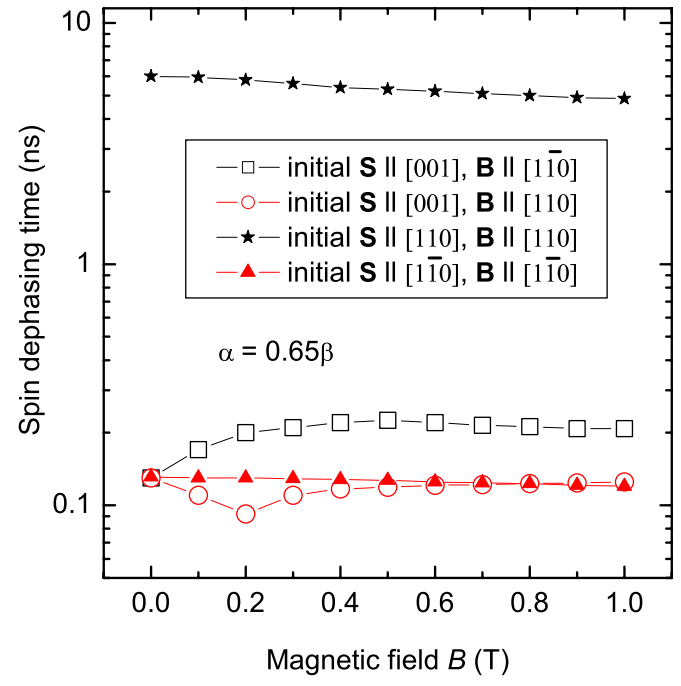

FIG. 4. (Color online) Comparison of spin-dephasing times, calculated with identical parameters for initial spin polarizations parallel to the [001] (open symbols) and the [110] and [1 $\overline{1} 0]$ directions (solid symbols), in dependence on magnetic fields parallel to the [110] and [1 $\overline{1} 0]$ in-plane directions.

Rashba coefficient, $\alpha=0.9 \mathrm{meV} \AA$, corresponds to a built-in electric field along the $z$ axis of $16.8 \mathrm{kV} / \mathrm{cm},{ }^{27}$ which is comparable with that of $15.0 \mathrm{kV} / \mathrm{cm}$, which we calculated from the heterostructure of our sample self-consistently. ${ }^{28}$ Using these parameters, the SDTs for spins initially aligned parallel to the magnetic field along the $[110]$ and $[1 \overline{1} 0]$ directions are calculated, ${ }^{29}$ as Fig. 4 shows. Here, we predict that the SDT for spins aligned parallel to [110] is several nanoseconds (black stars in Fig. 4), comparable to the value observed in the [110]-grown quantum wells. ${ }^{8}$

The observed phenomena can be well understood by the inhomogeneous broadening of the competing Dresselhaus and Rashba spin-orbit couplings. As the inhomogeneous

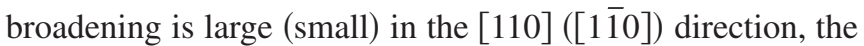
spin dephasing is large (small) for spin precession in the (110) $[(1 \overline{1} 0)]$ plane, i.e., along the $[110]([1 \overline{1} 0])$ axis. As the spin-orbit field is also strong, ${ }^{25}$ a small magnetic field cannot force all the spins to precess around it and there are spin precessions around the direction perpendicular to the magnetic field. Therefore, increasing the magnetic field suppresses the spin precessions along the direction perpendicular to the magnetic field and hence decreases (increases) the

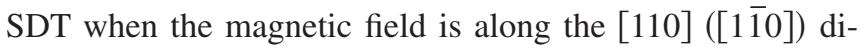
rection. On the other hand, if the magnetic field is further increased, it will drive the spins to tilt toward it in favor of the energy. Due to the anisotropy of the effective magnetic field, the inhomogeneous broadening decreases (increases)

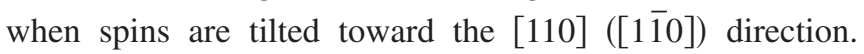
These two competing effects lead to the minimum (maximum) of the SDT observed in the experiment when the magnetic field is along [110] ([1 $\overline{1} 0])$. We note that measurements with a very high accuracy of alignment of the sample with respect to magnetic-field direction (less than $0.1^{\circ}$ misalignment) indicate that for the magnetic-field values applied in 
this work, there is no significant influence of a possible perpendicular field component due to slight misalignment on the SDT.

In summary, we have performed time-resolved Faraday rotation experiments on a high-mobility, [001]-grown 2DES. By applying a magnetic field in two different in-plane directions, we measure the in-plane anisotropy of the SDT. The measurements are compared to the fully microscopic calculations, and the comparison yields the strength of the Rashba spin-orbit coupling. Using these parameters, we calculate an in-plane SDT of several nanoseconds for our sample. We note that even though the TRFR experiment probes the outof-plane component of the spin polarization, it nevertheless demonstrates the in-plane spin-dephasing anisotropy and allows us to quantify it.

We gratefully acknowledge Jaroslav Fabian and R. T. Harley for valuable discussions. This work was supported by the Deutsche Forschungsgemeinschaft via GrK 638, Grants No. Schu1171/1-3, No. SFB 689 and No. SPP1285. M.W.W. was supported by the National Natural Science Foundation of China under Grant No. 10574120, the National Basic Research Program of China under Grant No. 2006CB922005, the Knowledge Innovation Project of the Chinese Academy of Sciences, and SRFDP. J.H.J. would like to thank J. Zhou for coding.

*mwwu@ustc.edu.cn

†christian.schueller@physik.uni-regensburg.de

${ }^{1}$ Semiconductor Spintronics and Quantum Computation, edited by D. D. Awschalom, D. Loss, and N. Samarth, Nanoscience and Technology (Springer, Berlin, 2002), and references therein.

${ }^{2}$ I. Zutic, J. Fabian, and S. Das Sarma, Rev. Mod. Phys. 76, 323 (2004), and references therein.

${ }^{3}$ S. Datta and B. Das, Appl. Phys. Lett. 56, 665 (1990).

${ }^{4}$ J. M. Kikkawa and D. D. Awschalom, Phys. Rev. Lett. 80, 4313 (1998).

${ }^{5}$ J. M. Kikkawa, I. P. Smorchkova, N. Samarth, and D. D. Awschalom, Science 277, 1284 (1997).

${ }^{6}$ A. Malinowski, R. S. Britton, T. Grevatt, R. T. Harley, D. A. Ritchie, and M. Y. Simmons, Phys. Rev. B 62, 13034 (2000).

${ }^{7}$ M. A. Brand, A. Malinowski, O. Z. Karimov, P. A. Marsden, R. T. Harley, A. J. Shields, D. Sanvitto, D. A. Ritchie, and M. Y. Simmons, Phys. Rev. Lett. 89, 236601 (2002).

${ }^{8}$ Y. Ohno, R. Terauchi, T. Adachi, F. Matsukura, and H. Ohno, Phys. Rev. Lett. 83, 4196 (1999).

${ }^{9}$ See also S. Döhrmann, D. Hägele, J. Rudolph, M. Bichler, D. Schuh, and M. Oestreich, Phys. Rev. Lett. 93, 147405 (2004).

${ }^{10}$ M. I. D'yakonov and V. I. Perel', Zh. Eksp. Teor. Fiz. 60, 1954 (1971) [Sov. Phys. JETP 33, 1053 (1971)].

${ }^{11}$ Y. A. Bychkov and E. Rashba, Zh. Eksp. Teor. Fiz. 39, 66 (1984) [Sov. Phys. JETP 39, 78 (1984)].

${ }^{12}$ G. Dresselhaus, Phys. Rev. 100, 580 (1955).

${ }^{13}$ N. S. Averkiev and L. E. Golub, Phys. Rev. B 60, 15582 (1999); M. M. Galzov, ibid. 70, 195314 (2004).

${ }^{14}$ Interestingly, it is demonstrated that the longest diffusion length does not occur at exactly $\alpha=\beta$ if the cubic term of the Dresselhaus term is included by J. L. Cheng, M. W. Wu, and I. C. da

Cunha Lima, Phys. Rev. B 75, 205328 (2007).

${ }^{15}$ J. Schliemann, J. C. Egues, and D. Loss, Phys. Rev. Lett. 90, 146801 (2003).

${ }^{16}$ N. S. Averkiev, L. E. Golub, A. S. Gurevich, V. P. Evtikhiev, V. P. Kochereshko, A. V. Platonov, A. S. Shkolnik, and Yu. P. Efimov, Phys. Rev. B 74, 033305 (2006).

${ }^{17}$ M. W. Wu and H. Metiu, Phys. Rev. B 61, 2945 (2000).

${ }^{18}$ M. Q. Weng and M. W. Wu, Phys. Rev. B 68, 075312 (2003).

${ }^{19}$ M. Q. Weng, M. W. Wu, and L. Jiang, Phys. Rev. B 69, 245320 (2004).

${ }^{20}$ J. Zhou, J. L. Cheng, and M. W. Wu, Phys. Rev. B 75, 045305 (2007).

${ }^{21}$ J. L. Cheng and M. W. Wu, J. Appl. Phys. 99, 083704 (2006).

${ }^{22}$ B. Liu, H. Zhao, J. Wang, L. Liu, W. Wang, D. Chen, and H. Zhu, Appl. Phys. Lett. 90, 112111 (2007).

${ }^{23}$ G. L. Bir, A. G. Aronov, and G. E. Pikus, Zh. Eksp. Teor. Fiz. 69, 1382 (1975) [Sov. Phys. JETP 42, 705 (1960)].

${ }^{24}$ J. Zhou and M. W. Wu, e-print arXiv:0705.0216 (unpublished).

${ }^{25}$ D. Stich, J. Zhou, T. Korn, R. Schulz, D. Schuh, W. Wegscheider, M. W. Wu, and C. Schüller, Phys. Rev. Lett. 98, 176401 (2007).

${ }^{26}$ Here $m_{c v}=0.667 m_{0}$.

${ }^{27}$ P. Pfeffer, Phys. Rev. B 59, 15902 (1999).

${ }^{28}$ P. Harrison, Quantum Wells, Wires and Dots: Theoretical and Computational Physics of Semiconductor Nanostructures (Wiley, New York, 2005), Chap. 3.

${ }^{29}$ Unlike the SDT due to the D'yakonov-Perel' mechanism calculated from the single-particle theory in the literature (Refs. 10 and 13) where a strong scattering limit is required, our spin kinetic Bloch equation approach is valid in both the strong and weak scattering limits; see also C. Lü, J. L. Cheng, and M. W. Wu, Phys. Rev. B 73, 125314 (2006). 